\title{
Brucelosis: prevalencia y factores de riesgo asociados en bovinos, bubalinos, caprinos y ovinos de Formosa, Argentina
}

\author{
Martinez, D.E. ${ }^{1}$; Cipolini, M.F. ; Storani, C.A. ${ }^{1}$; Russo, A.M. ${ }^{2}$; Martinez, E.I. ${ }^{1}$ \\ ${ }^{1}$ Cátedra Enfermedades Infecciosas, Fac. Cs. Veterinarias, Univ. Nac. Nordeste (UNNE), Sargento Cabral 2139, \\ Corrientes 3400, Argentina. ${ }^{2}$ Centro de Investigación y Transferencia (CIT) Formosa, Ruta 11, km 1164, \\ 3600 Formosa, Argentina.
}

\begin{abstract}
Resumen
Martinez, D.E.; Cipolini, M.F.; Storani, C.A.; Russo, A.M.; Martinez, E.I.: Brucelosis: prevalencia y factores de riesgo asociados en bovinos, bubalinos, caprinos y ovinos de Formosa, Argentina. Rev. vet. 29: 1, 40-44, 2018. La brucelosis es una enfermedad infectocontagiosa que afecta a especies domésticas y salvajes, caracterizada por la producción de trastornos reproductivos y pérdidas en la producción. Es además una zoonosis de importancia mundial. Existen factores de riesgo asociados a la presencia de brucelosis en los animales domésticos, que favorecen el ingreso y la permanencia de la enfermedad en una población. En el presente trabajo se realizó un análisis de la prevalencia serológica de la brucelosis en rodeos bovinos, bubalinos, caprinos y ovinos, así como la correlación entre la presencia de animales serorreactores y determinados factores de riesgo. El trabajo se realizó durante 3 años en establecimientos pecuarios de la Provincia de Formosa. Se compararon encuestas epidemiológicas con la información proveniente de muestreos para análisis serológicos. Se investigó un total de 1686 animales, de los cuales 657 fueron bovinos, 265 bubalinos, 569 caprinos y 195 ovinos. Se analizaron 40 establecimientos pecuarios de los departamentos Formosa, Pilagá, Pirané, Pilcomayo y Patiño. La prevalencia de rodeos positivos fue de 12,5; 50; 40,9 y $0 \%$ y de animales positivos de 1,$37 ; 10,94 ; 7,9$ y $0 \%$ en bovinos, bubalinos, caprinos y ovinos respectivamente. El análisis de odds ratio arrojó significación estadística para los factores: (a) presencia de síntomas, (b) aislamiento de hembras próximas al parto, (c) cobertura vacunal y (d) tamaño del rodeo. El estadístico chi cuadrado mostró diferencias significativas en la presentación de brucelosis comparando bovinos con bubalinos y caprinos, pero no hubo diferencias cuando se compararon bubalinos y caprinos entre sí. Se concluye que la brucelosis está presente en los rodeos de animales domésticos de la Provincia de Formosa, que existen factores de riesgo asociados al mantenimiento de la enfermedad en las poblaciones y que la aplicación regular de medidas de manejo sanitario reduce la frecuencia de la enfermedad.
\end{abstract}

Palabras clave: rumiantes, brucelosis, epidemiología, norte argentino, prevalencia.

\begin{abstract}
Martinez, D.E.; Cipolini, M.F.; Storani, C.A.; Russo, A.M.; Martinez, E.I.: Brucellosis: prevalence and associated risk factors in cattle, buffalo, goats and sheep from Formosa, Argentina. Rev. vet. 29: 1, 40-44, 2018. Brucellosis is an infectious disease affecting domestic and wild species, characterized by the production of reproductive disorders and production losses. It is also a zoonosis of global importance. There are risk factors associated with the presence of brucellosis in domestic animals, which favor the entry and permanence of the disease in a population. In the present work an analysis of the serological prevalence of brucellosis in bovine, buffalo, goat and sheep herds was performed and the correlation between the presence of positive animals and certain risk factors. The work was carried out during 3 years in livestock establishments of the Province of Formosa. Information from samples for serological analysis and epidemiological surveys was compared. A total of 1686 animals were investigated, of which 657 were cattle, 265 buffalo, 569 goats and 195 sheep. Forty livestock farms in the departments of Formosa, Pilagá, Pirané, Pilcomayo and Patiño were analyzed. The prevalence of positive herds was $12.5 ; 50 ; 40.9$ and $0 \%$; prevalence of positive animals were $1.37 ; 10.94 ; 7.9$ and $0 \%$ in cattle, buffaloes, goats and sheep respectively. The Odds Ratio analysis showed statistical significance for the factors: presence of symptoms, isolation of females close to calving, vaccination coverage and size of the herd. The $X^{2}$ statistic showed significant differences in the presentation of brucellosis when comparing cattle with buffaloes and goats, but there were no differences when buffaloes and goats
\end{abstract}


were compared. It is concluded that brucellosis is present in the herds of domestic animals of the Province of Formosa and there are risk factors associated with the maintenance of the disease in the populations. Regular application of health management measures reduces the frequency of disease in domestic animals.

Key words: ruminants, brucellosis, epidemiology, north Argentinean, prevalence.

\section{INTRODUCCIÓN}

La brucelosis es una enfermedad infecto-contagiosa que afecta a especies domésticas de explotación tradicional -bovinos, caprinos, ovinos-y otros animales no tradicionales como el búfalo. Se caracteriza por la producción de abortos en el último tercio de gestación, retención de placenta, metritis, infertilidad, natimortos, mastitis, menor producción y calidad de leche, afecciones articulares, orquitis y epididimitis, constituyéndose en una zoonosis ocupacional que afecta con más frecuencia a los veterinarios y trabajadores rurales, así como personas vinculadas a la cadena productiva ${ }^{5,11,21}$.

Entre las enfermedades que afectan a la producción, la brucelosis constituye una de las más importantes, por el gran impacto económico que tiene sobre los índices de productividad de los rodeos ${ }^{5,21}$. Está descripta una serie de factores de riesgo asociados a la presencia de brucelosis en los rodeos de animales domésticos, que se comportan de tal modo por favorecer el ingreso y la permanencia de la enfermedad en una población.

Entre ellos se pueden citar la incorporación de animales de los cuales se desconoce la situación epizootiológica y la cantidad de los mismos que integran el rodeo, registrándose un aumento en el riesgo de infección en relación directa con la cantidad de animales coexistiendo en un mismo hato y la convivencia entre especies, tanto domésticas como salvajes, ya que algunas se comportan como reservorios.

Se han señalado a los perros como diseminadores de la enfermedad al momento de ingerir placentas y/o fetos nacidos de hembras con brucelosis, pudiendo además ser reservorios del germen en sus propios tejidos ${ }^{2}$, $3,6,17,20$. Los cerdos y suinos salvajes han sido descriptos como potenciales reservorios de la enfermedad para los bovinos con quienes conviven ${ }^{12}$. Los equinos pueden mantener el germen cuando padecen de la patología denominada "mal de la cruz", infección localizada en su ligamento nucal ${ }^{15}$.

Dentro de los eventos relacionados con medidas de manejo sanitario, la no remoción de los desechos de abortos y partos y la no eliminación de seroreactores positivos en un rebaño, han sido sindicados como factores de riesgo ${ }^{13}$. La presencia de animales vacunados se comporta como un factor protector, aunque se ha demostrado que el $10 \%$ de éstos puede diseminar asimismo el germen causal ${ }^{19}$.

El registro de síntomas clínicos de la enfermedad en los animales de un rebaño, indica que se ha quebrado el equilibrio entre agente y huésped, y también está relacionado con el aumento de infectados en esa población ${ }^{7}$. Cuando las distintas especies comparten aguadas y pastizales contaminados con secreciones de hembras infectadas, se incrementan las posibilidades de contagio ${ }^{8,16}$. La bacteria se mantiene por ciertos períodos de tiempo en los corrales y establos donde las hembras paren o abortan ${ }^{14}$.

La no eliminación de las hijas de madres positivas incrementa las posibilidades de que permanezcan en el rodeo hembras que se han infectado durante la gestación o en el momento de atravesar el canal del parto. La mayoría de las hembras así nacidas se libera de la infección, pero un porcentaje de ellas permanece infectado hasta la edad adulta, momento en que la bacteria será reactivada con la primera gestación ${ }^{18,23,25}$. La transhumancia - habitual en pequeños rumiantes- propicia que rebaños de diversos productores compartan pastizales y aguadas, y que transmitan la bacteria entre sus integrantes ${ }^{1}$.

En el presente trabajo se realizó un análisis de la prevalencia serológica de la brucelosis en rodeos bovinos, bubalinos, caprinos y ovinos y se estudió la correlación entre la presencia de animales serorreactores y determinados factores de riesgo asociados al ingreso y manutención de la enfermedad en los rodeos.

\section{MATERIAL Y MÉTODOS}

La investigación se realizó a lo largo de 3 años en establecimientos pecuarios de la Provincia de Formosa. Se comparó información proveniente de muestreos para análisis serológicos y encuestas epidemiológicas orientadas a la identificación de factores de riesgo para la presencia de brucelosis.

Se trabajó con un total de 1686 animales, de los cuales 657 fueron bovinos, 265 bubalinos, 569 caprinos y 195 ovinos. Se analizaron 40 establecimientos pecuarios de los departamentos Formosa, Pilagá, Pirané, Pilcomayo y Patiño de la mencionada provincia. Para la estimación de prevalencias, se determinó la cantidad de animales a analizar en función del tamaño de los rodeos en cada establecimiento y especie, así como la prevalencia esperada, siguiendo fórmulas diseñadas para tal fin ${ }^{24}$.

Se tomaron muestras de sangre entera para la obtención de suero en bovinos, búfalos, cabras y ovejas de los establecimientos seleccionados. Los sueros se analizaron para brucelosis utilizando las técnicas de Antígeno Bufferado en Placa (BPA) como tamiz y las de Fijación del Complemento (FC) y Polarización Fluo- 
rescente (FPA) como confirmatorias, siguiendo la normativa vigente en el país ${ }^{22}$.

Para estudiar factores de riesgo se tomaron en cuenta los datos recolectados en 36 encuestas epidemiológicas. Se consideraron rodeos positivos a aquellos que tenían al menos un animal seropositivo integrándolo. Se realizó encuesta epidemiológica para cada una de las especies que convivían en cada establecimiento.

La encuesta contempló factores intrínsecos y extrínsecos a los rebaños que pudieran favorecer la presencia y manutención de la brucelosis en los grupos de animales. Se asignó un código y una clasificación numérica a cada alternativa, para luego poder analizar los resultados cualitativos.

Los análisis estadísticos realizados fueron la estimación de la prevalencia de brucelosis en cada especie y se utilizaron chi cuadrado $\left(X^{2}\right)$ y odds ratio $(\mathrm{OR})$ para la comparación de eventos epidemiológicos ${ }^{4,24}$.

En cada rodeo se llevó a cabo el estudio de la relación existente entre las variables presencia/ausencia de seropositivos y los siguientes factores de riesgo: a) existencia/ausencia de animales con síntomas compatibles con la enfermedad; b) manejo de las hembras de la reposición y las que están próximas a parir expresadas como presencialausencia de reposición con hijas de madres positivas; c) cobertura vacunal (en las dos especies que se vacunan, bovinos y bubalinos) expresada como rodeos con $80 \%$ o más de animales vacunados y rodeos con menos de $80 \%$ de animales vacunados, y d) tamaño del rodeo, expresados en rodeos pequeños/ grandes variando el límite en cada especie: para bovinos y bubalinos el punto de corte fue de 250 animales totales y para ovinos y caprinos de 50 animales.

Se realizaron cuadros de doble entrada para efectuar los cálculos de odds ratio -a fin de estimar el riesgo relativo de que ocurra un evento-además del valor $p$ en cada caso, que establece la significación estadística (cuando $\mathrm{p} \leq 0,05$ ).

Fueron incluidos en el estudio otros factores de riesgo, pero no se realizaron comparaciones al detectar luego del análisis de las encuestas que todos los rodeos los compartían en igual medida.

\section{RESULTADOS Y DISCUSIÓN}

En la Tabla 1 se muestra la prevalencia de serorreactores positivos a brucelosis, discriminados por especie.

Se observa que la proporción de rodeos positivos y la prevalencia individual son elevadas en las especies

Tabla 1. Serorreactores positivos a brucelosis según especie y prevalencias individuales y por rodeos.

\begin{tabular}{lcccc}
\hline \multirow{2}{*}{ especie } & \multicolumn{2}{c}{ prevalencia por rodeos } & \multicolumn{2}{c}{ prevalencia individual } \\
\cline { 2 - 5 } & positivos/total & porcentaje & positivos/total & porcentaje \\
\hline bovinos & $3 / 24$ & $12,5 \%$ & $9 / 657$ & $1,37 \%$ \\
bubalinos & $2 / 4$ & $50 \%$ & $29 / 265$ & $10,94 \%$ \\
caprinos & $9 / 22$ & $40,9 \%$ & $45 / 569$ & $7,9 \%$ \\
ovinos & $0 / 14$ & $0 \%$ & $0 / 195$ & $0 \%$ \\
\hline
\end{tabular}

bubalina y caprina. Los bubalinos están incluidos en el Plan Nacional de Control y Erradicación de la Brucelosis desde hace algunos años ${ }^{22}$, un lapso menor de tiempo si se compara con los bovinos, lo que podría evidenciarse en la cantidad de positivos que exhibe cada especie.

Otro aspecto a considerar para la implementación de las medidas sanitarias está representado por la manera en que los bubalinos son explotados, en condiciones extensivas y en terrenos bajos y anegadizos, donde no siempre se respetan los tiempos de vacunación y esto podría conducir a la persistencia de anticuerpos vacunales, resultando en falsos positivos cuando los animales son examinados en la edad adulta.

En cuanto a los caprinos, es una especie que se explota mayormente por comunidades vulnerables, de escasos recursos y con insuficiente acceso al asesoramiento profesional para la prevención de enfermedades, lo cual sumado a que no está indicada por el ente sanitario la vacunación en la provincia, facilita el ingreso y permanencia de la brucelosis en los hatos.

Los bovinos, como se ha dicho, están desde hace décadas incorporados en planes de control y erradicación, lo que se refleja en la menor cantidad de animales serorreactores en los rodeos. Por último, el ovino es afectado primordialmente por una Brucella de cepa rugosa, $B$. ovis, y puede además ser contagiado por $B$. melitensis, una cepa lisa cuyo principal hospedador es la cabra ${ }^{9}$.

Los análisis efectuados en el presente trabajo están orientados a detectar esta última especie de Brucella, evidenciándose, al menos en los hatos estudiados, que no habría transmisión de $B$. melitensis entre cabras y ovejas en las condiciones evaluadas, dado que en el mismo establecimiento pecuario convivían rodeos de caprinos positivos con ovinos negativos.

En la Tabla 2 se muestran los resultados del análisis de la razón de proporciones-odds ratio-discriminados por especie. No se incluyen resultados en ovinos dado que no hubo serorreactores positivos en esta especie.

La presencia de animales con síntomas de brucelosis representó un factor de riesgo de relevancia sólo para la especie caprina. Esto podría deberse a que las otras dos especies donde hubo positivos -bovinos y búfalos- son vacunados sistemáticamente por estar incluida esta actividad de manera obligatoria dentro del plan de control que los afecta.

Es sabido que una hembra, si está vacunada contra brucelosis, aún estando infectada eliminará menos cantidad de bacterias al medio ambiente, reduciendo así las posibilidades de contagio ${ }^{10}$. Las cabras, como se ha dicho, no se vacunan en la región en estudio.

El factor de riesgo que contempla el asilamiento o no de las hembras próximas a parir, que incide directamente en la presencia de bacterias en el ambiente cuando éstas son eliminadas con los abortos o partos normales de hembras brucelosas, también fue estadísticamente significativo en las cabras y podría 
Tabla 2. $O R$ y valor $p$ para los factores de riesgo analizados, discriminados por especie en la Provincia de Formosa.

\begin{tabular}{lccccccc}
\hline \multirow{2}{*}{ factor de riesgo } & \multicolumn{2}{c}{ bovinos } & \multicolumn{2}{c}{ bubalinos } & \multicolumn{2}{c}{ caprinos } \\
& OR & $\mathrm{p}$ & OR & $\mathrm{p}$ & OR & $\mathrm{p}$ \\
\hline presencia de síntomas & 1,32 & 0,80 & 1,11 & 0,92 & 4,62 & $0,0041^{*}$ \\
aislamiento de hembras & 1,71 & 0,64 & 9,37 & 0,12 & 0,36 & $0,0012^{*}$ \\
cobertura vacunal & 0,80 & 0,88 & 0,04 & $<0,0001^{*}$ & - & - \\
tamaño del rodeo & 0,45 & 0,49 & 0,072 & $<0,0001^{*}$ & 0,63 & 0,14 \\
\hline
\end{tabular}

OR: odds ratio, p: significación. *Celdas marcadas indican significación estadística.

Tabla 3. Análisis de chi cuadrado $\left(X^{2}\right)$ para la distribución de serorreactores positivos en función de las especies donde éstos fueron detectados.

\begin{tabular}{lcc}
\hline especies analizadas & valor $X^{2}$ & valor $p$ \\
\hline bovinos/bubalinos & 38,88 & $<0,0001^{*}$ \\
bovinos/caprinos & 28,25 & $<0,0001^{*}$ \\
bubalinos/caprinos & 1,70 & 0,19 \\
\hline
\end{tabular}

*celdas marcadas indican significación estadística

relacionarse directamente con la explicación vertida para el factor de riesgo anterior.

La presencia de $80 \%$ o más de animales vacunados en los rodeos fue significante como factor protector para el ganado bubalino y esto podría relacionarse con las prevalencias halladas, que son mayores en esta especie que en el bovino. Al haber posiblemente más animales infectados en los rodeos bubalinos, una mayor cobertura vacunal incidiría directamente en la tasa de contagios. En los bovinos, en cambio, al ser menor la proporción de animales afectados, también se reduciría la tasa de contagio, aunque las coberturas vacunales no sean óptimas.

Con respecto al tamaño del rodeo, sólo en los hatos bubalinos representó un factor de riesgo que influiría en la permanencia de la enfermedad, favoreciendo que ésta se halle presente en mayor medida en los rodeos más numerosos. Ello podría deberse a que los rebaños de mayor tamaño a menudo incorporan animales a sus existencias, a través de la compra de reproductores que, si no están debidamente controlados, pueden ingresar la enfermedad. Los rodeos pequeños son a menudo cerrados y la reposición de reproductores es realizada con los propios animales.

En la tabla 3 se muestran los resultados de la prueba de chi cuadrado. El análisis de la distribución de los positivos cuando se compararon a las especies donde hubo serorreactores entre sí, reveló la existencia de una significativa diferencia al cotejar a bovinos con bubalinos y caprinos, indicando que hubo asociación entre las variables especie y positividad a brucelosis, siendo los bovinos los menos expuestos. Esto no ocurrió, sin embrago, al comparar los resultados de bubalinos y caprinos, especies donde hubo una alta proporción de reactores a brucelosis.

Cabe destacar que hubo otros factores de riesgo que no se incluyeron en el análisis, ya que afectaban a todos los rodeos por igual, como la incorporación de animales sin controles serológicos previos, el contacto con animales silvestres y la presencia de zonas geográficas inundables, entre otros.

Se concluye que la brucelosis estuvo presente en los rodeos de animales domésticos estudiados en la Provincia de Formosa, excepto en ovinos, bajo las condiciones del estudio. Bubalinos y caprinos fueron los más afectados, seguidos por los bovinos. Dentro de las poblaciones existen factores de riesgo asociados al mantenimiento de la afección. La aplicación regular de medidas de manejo sanitario de los animales reduce la frecuencia de la enfermedad.

Agradecimientos. A los becarios y ayudantes alumnos de la Cátedra de Enfermedades Infecciosas (DCV-UNNE) por su colaboración en los trabajos de campo y en la sistematización de los datos.

\section{REFERENCIAS}

1. Abernethy DA et al. 2011. Epidemiology and management of a bovine brucellosis cluster in Northern Ireland. Prev Vet Med 98: 223-229.

2. Aldiri G, Llorentes F, Silveira E. 1992. Comportamiento de la evolución de la brucelosis en cooperativas de diferentes tamaños vacunadas y sin vacunar. Rev Cub Cienc Vet 23: 117-122.

3. Baek BK, Lim CW, Rahman MS, Kim CH, Oluoch A, Kakoma I. 2003. Brucella abortus infection in indigenous Korean dogs. Can J Vet Res 67: 312-314.

4. Bland JM, Altman DG. 2000. The odds ratio. British Med J 320: 7247, p. 1468.

5. Blood DC, Radostits OM. 1992. Enfermedades causadas por bacterias. En: Medicina Veterinaria, $7^{\circ}$ ed., McGrawHill Interamericana, New York, p. 720-742.

6. Clavijo E, Ramos P, Chantada R. 1976. Reporte del aislamiento de una cepa de Brucella suis del testículo de un perro. En: Resúmenes del II Congreso Nacional de Ciencias Veterinarias, La Habana, Cuba.

7. Fernández A. 1982. Algunos aspectos epizootiológicos de la brucelosis bovina en las condiciones de la República de Cuba. Tesis doctoral. Escuela Superior de Veterinaria de Brno, Checoslovaquia, p. 15-38.

8. Fosgate GT, Diptee MD, Ramnanan A, Adesiyun AA. 2011. Brucellosis in domestic water buffalo (Bubalus bubalis) of Trinidad and Tobago with comparative epidemiology to cattle. Trop Anim Health Prod 43: 1479-1486.

9. Garin BB, Blasco JM, Grayon M, Verger JM. 1998. Brucella melitensis infection in sheep: present and future. Veterinary Res 29: 255-274.

10. Lord VR, Schurig GG, Cherwonogrodzky JW, Marcano MJ, Melendez GE. 1998. Field study of vaccination of cattle with Brucella abortus strains RB51 and 19 under high and low disease prevalence. Am J Vet Res 59: 10161020. 
11. Lüchter F. 2003. Enfermedades infecciosas de los rumiantes. En: Introducción al estudio de las enfermedades infecciosas, 1ra.ed., Ed. Colorgrafic, Buenos Aires, Argentina, p. 175.

12. Martínez E, Valdes J, Roman J, Rodríguez L. 1989. Ecosistema de brucelosis animal en el municipio de Consolación del Sur, Provincia de Pinar del Río, Cuba. Rev Cienc y Téc Agric Vet 11: 17-24.

13. Moreno R, Rentería E, Searcy B. 2002. Seroprevalencia y factores de riesgos asociados a la brucelosis bovina en hatos lecheros de Tijuana, Baja California. Rev Téc Pec Méx 40: 243-249.

14. Muma JB, Godfroid J, Samui KL, Skjerve E. 2007. The role of Brucella infection in abortions among traditional cattle reared in proximity to wildlife on the Kafue, Zambia. Rev Sci Tech Off Int Epiz 26: 721-730.

15. Ocholi RA, Bertu WJ, Kwaga JK, Ajogi I, Bale JO, Okpara J. 2004. Carpal bursitis associated with Brucella abortus in a horse in Nigeria. Vet Rec 155: 566-567.

16. Organización Mundial de Sanidad Animal (OIE). 2010. Bovine brucellosis, Chapter 2.4.3. In: Manual of Diagnostic Tests and Vaccines for Terrestrial Animals, OIE, París, p. 1-35.

17. Pereira L, Gómez M, Jaime M. 1999. Aspectos epidemiológicos de la brucelosis ovina en la Provincia de León. Anales VIII Jornada Producción Animal, León, España, p. 378-380.
18. Plommet M, Renoux G, Philipon A, Gestin J, Fensterbank R. 1971. Congenital transmission of bovine brucellosis from one generation to another. Bull Acad Vét Fr 44 : 5-59.

19. Rogerson B, Morgan I. 1989. Investigaciones de reacciones positivas aberrantes a pruebas serológicas para la brucelosis bovina. Información Express: Serie Veterinaria 13: 1-65, p. 30.

20. Salazar A, Moreno J, Ramírez I. 2002. Aislamiento de Brucella suis biotipo 1. Memorias del XVIII Congreso Panamericano de Ciencias Veterinarias, La Habana, Cuba, CD-ROM: 7G 4-2-3 0203271505-963127.

21. Seifert HS. 1998. Enfermedades transmisibles por contagio. En: Sanidad Animal en los Trópicos, $1^{\mathrm{a}}$ ed., Edit. Hemisferio Sur, Buenos Aires, Argentina, p. 268-278.

22. Servicio Nacional de Sanidad y Calidad Agroalimentaria (SENASA). Plan nacional de control y erradicación de la brucelosis bovina. Resoluciones 150/2002, 725/2005.

23. Terhuurne AA, Meijer M, Dijkerman NA. 1993. Latency of Brucella abortus causes problems in oriented control: a review. Tijdschr Diergeneeskd 118: 679-683.

24. Thrusfield M. 1990. Epidemiología Veterinaria, Editorial Acribia, Zaragoza, España,. págs. 42, 180-185, 196-204.

25. Wilesmith JW. 1978. The persistence of B. abortus infection in calves. A retrospective study of heavily infected herd. Vet Rec 103: 149-153.

\section{Revista Veterinaria obtuvo el máximo nivel de categorización del CAICYT-CONICET}

Tras el pertinente proceso de evaluación según criterios de calidad editorial, en setiembre de 2005 CAICYT-CONICET ha clasificado a nuestra publicación con Categoría 1 (nivel superior de excelencia), con lo cual pasa a integrar el Catálogo Latindex (folio 14022). La Dirección de Revista veterinaria agradece a quienes colaboraron para obtener tan importante distinción. Ver: http://www.latindex. unam.mx/busquedas/catalogotitulo.html 